\title{
Supera self-expanding stents for endovascular treatment of femoropopliteal disease: a review of the clinical evidence
}

This article was published in the following Dove Press journal:

Vascular Health and Risk Management

13 July 2015

Number of times this article has been viewed

\author{
Kalkidan Bishu' ${ }^{1,2}$ \\ Ehrin J Armstrong ${ }^{1,2}$ \\ 'Division of Cardiology, University of \\ Colorado, Aurora, ${ }^{2}$ Denver VA Medical \\ Center, Denver, CO, USA
}

\begin{abstract}
Femoropopliteal lesions account for a significant proportion of endovascular interventions for peripheral artery disease in patients with disabling claudication or chronic limb ischemia. The femoropopliteal artery crosses two joint structures (hip and knee joints) and courses through the muscular adductor canal in the thigh, which places the artery at increased biomechanical stress. There is a critical need for stent platforms with a reduced risk of stent fracture while maintaining patency during long-term follow-up. The Supera peripheral stent system has a braided nickel-titanium alloy stent designed to withstand the unique stressors along the course of the femoropopliteal artery. This design may be associated with improved patency in association with reduced stent fracture rates on short- and medium-term follow-up. Further studies, including randomized controlled studies, comparing the Supera interwoven nickel-titanium alloy stent system with other stent platforms and angioplasty alone are needed.

Keywords: peripheral artery disease, femoropopliteal atherosclerosis, SUPERA interwoven nitinol stent, stent fracture
\end{abstract}

\section{Introduction}

Approximately 8.5 million American adults are affected by peripheral arterial disease. Endovascular intervention is indicated in the setting of disabling claudication despite optimal medical therapy or critical limb ischemia with ischemic rest pain or ischemic tissue loss..$^{1-3}$ There has been a marked increase in the utilization of endovascular interventions in the treatment of peripheral arterial disease, with femoropopliteal interventions accounting for more than $55 \%$ of cases. ${ }^{4}$ The femoropopliteal artery crosses two joint structures (hip and knee joints) and courses through the muscular adductor canal in the thigh, which places the artery at increased stress from torsion, bending as well as longitudinal and radial compression forces. Stents in the femoropopliteal system have historically been associated with increased rates of stent fracture, which is associated with high rates of restenosis and decreased primary patency rate during long-term follow-up. ${ }^{5-8}$ There is, therefore, a critical need for stent platforms with minimal-to-no risk of stent fracture.

The Supera peripheral stent system is a braided nickel-titanium alloy (nitinol) stent that was designed to withstand the unique stressors along the course of the femoropopliteal artery. In this manuscript, we describe the Supera peripheral stent system and review the available clinical data regarding its application to endovascular treatment of femoropopliteal disease. We also review the literature on application of the Supera stent system to specific clinically important lesion subsets, including popliteal artery disease, heavily calcified lesions, and long femoropopliteal lesions.
Correspondence: Ehrin J Armstrons

Denver VA Medical Center,

1055 Clermont Street, Denver,

CO 80220, USA

Email ehrin.armstrong@gmail.com 


\section{Overview of the Supera peripheral stent system}

The Supera peripheral stent is a woven self-expanding stent constructed from nitinol. ${ }^{9}$ Six pairs of closed-ended interwoven nitinol wires are arranged in a helical pattern designed to be both flexible and resistant to fracture. The stent comes premounted on an over-the-wire delivery system (6F or 7F) that is compatible with $0.014^{\prime \prime}$ or $0.018^{\prime \prime}$ guide wires. It is manufactured in $4.5 \mathrm{~mm}, 5.5 \mathrm{~mm}$, and $6.5 \mathrm{~mm}$ diameters, which correspond to the outer stent diameter with nominal stent lengths ranging from $20 \mathrm{~mm}$ to $150 \mathrm{~mm}$. Labeled stent diameters are chosen to match the reference vessel luminal diameter in a 1:1 ratio. The stent delivery system comes with a stent driver that brings the distal end of the stent into contact with the target vessel. Further manipulation deploys the stent out of the sheath as the delivery catheter moves proximally. Unlocking the deployment lock allows a final deployment stroke to detach the stent from the delivery catheter. Because of this unique deployment system, the stent can be stacked in certain lesion locations. Care must also be taken not to elongate the stent during deployment, as excessive elongation may be associated with less compression resistance and potentially a lower rate of primary patency. The Supera stent generally foreshortens during deployment, because the stent is three times longer within the catheter prior to deployment.

\section{Clinical outcomes of Supera stents in femoropopliteal disease}

A number of registry studies have examined the outcomes of Supera stents in the treatment of femoropopliteal disease. While none of these studies are randomized, the data regarding Supera stents can be compared to similar studies of other stent types (Tables 1 and 2).

The first retrospective study (Leipzig Supera Superficial Femoral Artery [SFA] Registry) of the use of Supera stents in atherosclerotic femoropopliteal lesion was reported by Scheinert et al. One hundred and seven lesions with Supera stents implanted in the femoropopliteal segments from 2007 to 2008 at Park Hospital in Leipzig, Germany, were studied. ${ }^{10}$ The mean stent length was $111 \mathrm{~mm}$. One-year and 2-year primary patency rates were $85 \%$ and $76 \%$, respectively. The mean ankle brachial index (ABI) increased from 0.68 to 0.87 and mean Rutherford-Becker class decreased from 3.3 to 2.0. No stent fractures were observed on follow-up radiography.

The SUPERB (comparison of the supera peripheral system to a performance goal derived from balloon angioplasty clinical in the superficial femoral artery) was a prospective multicenter study that included 34 participating sites in the United States enrolling 264 patients with femoropopliteal lesions, including popliteal above-knee lesions from July 2009 to $2011 .^{11}$ The mean lesion length in the SUPERB trial was $78 \mathrm{~mm}$. A total of $28 \%$ of lesions had moderate calcification and $45 \%$ had severe calcification. The primary patency rate at 1 year (the freedom from $>50 \%$ stenosis with peak systolic velocity [PSV] ratio $\leq 2.0$ on duplex ultrasound) was $79 \%$. Using a modified PSV criteria of $<2.4$, the primary patency rate at 12 months was $80 \% .^{9}$ The freedom from target lesion revascularization at 12 months was $89 \%$, and the ankle brachial index increased from 0.7 to 0.92 at 12 months. At 12-month follow-up, there was no evidence of stent fracture, whereas at 24 months, one patient had a stent fracture. ${ }^{9}$

In an update of their earlier results (Leipzig Supera 500 registry) of the use of Supera stents in femoropopliteal disease, ${ }^{12}$ Werner et al from Park Hospital in Leipzig followed 492 limbs (439 patients) treated with Supera stents between 2007 and 2011, of whom 53\% had total occlusion and 52\% had moderate or severe calcification. ${ }^{12}$ The mean lesion length was $126 \mathrm{~mm}$. Primary patency rates were $83 \%$ at 12 months and $73 \%$ at 2 years. Among lesions $\geq 150 \mathrm{~mm}$ in length (172 lesions, $33 \%$ of cases in this report), primary patency rates at 12 months and 24 months were $81 \%$ and $62 \%$, respectively. The mean ABI increased from 0.53 to 0.91 , and the mean Rutherford-Becker class decreased from 3.0 to 1.9. No stent fractures were observed at follow-up.

Other investigators have also confirmed the favorable outcomes for femoropopliteal stenting with the Supera stent. In 42 limbs (36 patients) treated with the Supera, 50\% had occlusions, $64 \%$ had moderate or severe calcification, with a mean lesion length of $105 \mathrm{~mm}$ and the primary patency rate at 12 months of $86 \%$, while ABI increased from 0.57 to 0.91 . There were no stent fractures. ${ }^{13}$

Chan et al also reported results from Supera placement in 78 patients with femoropopliteal mean lesion lengths of $126 \mathrm{~mm}$ at the University of Hong Kong Medical Center from October 2011 to April 2013. ${ }^{14}$ The primary patency rate at 12 months was $79 \%$, and ankle brachial index increased from 0.58 to 0.87 . No stent fractures were found.

George et al reported on 98 limbs in 80 patients with femoropopliteal lesions treated with Supera stents in the Supera interwoven nitinol stent outcomes in above-knee interventions study. ${ }^{15}$ The mean lesion length was $143 \mathrm{~mm}$, $38 \%$ of lesions were occlusive, and $22 \%$ of lesions involved the popliteal artery alone or extended into the popliteal artery. The mean ABI increased from 0.60 to 0.83 at last 

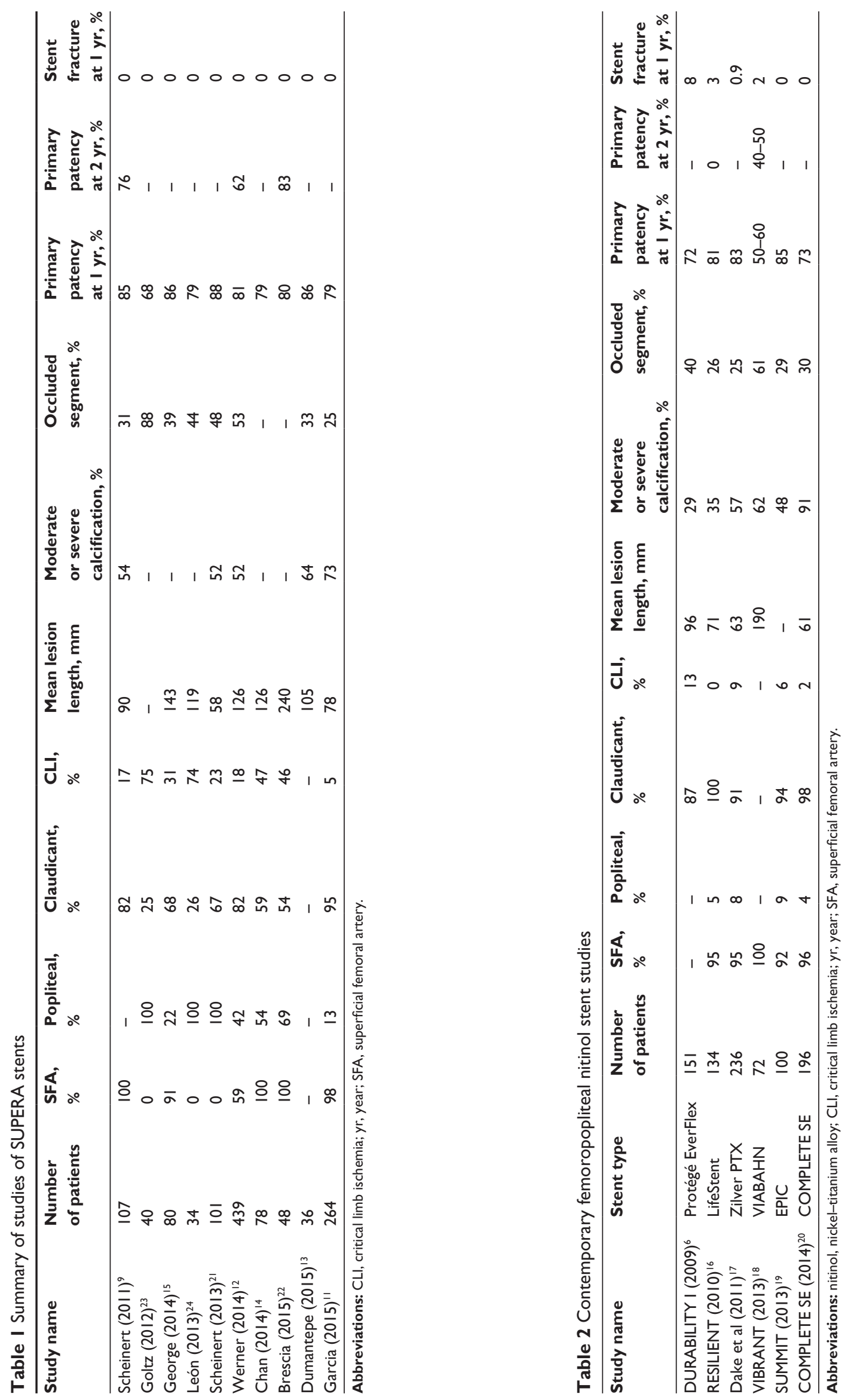
follow-up. The primary patency rate (based on the freedom from clinically driven target lesion revascularization and not ultrasound criteria) at 12 months was $86 \%$. Reintervention was required in 15 limbs (13 patients) by 12 months. No stent fractures were noted in patients who required reintervention.

\section{Comparison of Supera with other contemporary stent platforms}

While there have not been any studies directly comparing various nitinol self-expanding stent platforms in the femoropopliteal segment, the results described in Table 1 compare favorably to published reports of a selection of other contemporary stent types in the same anatomic segment (Table 2).

In the RESILIENT randomized trial, Laird et al randomized 206 patients with superficial femoral and proximal popliteal atherosclerotic disease to primary implantation of the LifeStent nitinol stent (Bard Peripheral Vascular, Inc., Tempe, AZ, USA) compared to percutaneous transluminal angioplasty with provisional stent implantation. ${ }^{16}$ At 12 months, the freedom from target lesion revascularization was $87 \%$ in the primary stenting group compared to $45 \%$ in the percutaneous transluminal angioplasty (PTA) group (bail-out stenting was considered target lesion revascularization due to loss of primary patency). Duplex US-derived primary patency at 12 months was $81 \%$ in the primary stent group compared to $37 \%$ in the PTA group. Stent fracture was observed in $3.1 \%$ of stents. A total of $40 \%$ of lesions in the PTA group required bail-out stenting due to inadequate PTA result, flow-limiting dissection, or residual stenosis $>30 \%$.

In a study of 151 patients with femoropopliteal artery disease treated with the Protégé EverFlex (ev3 Inc., Plymouth, MN, USA) self-expanding nitinol stent system (DURABILITY I) study mean lesion length was $96 \mathrm{~mm} .^{6}$ The primary patency rate at 1 year was $72 \%$. Stent fracture was noted in $8 \%$ of patients.

Dake et al compared the use of the Zilver (Cook Medical, Bloomington, IN, USA) paclitaxel-coated nitinol drugeluting stent (236 patients) to PTA with provisional drug eluting stent (DES) or bare metal stent (BMS) placement in acute PTA failure (238 patients out of which 120 patients underwent provisional stenting with BMS:DES of 1:1 ratio at randomization) in patients with femoropopliteal atherosclerosis. ${ }^{17}$ Seven percent of patients had lesions involving the popliteal artery. The mean lesion length was $63 \mathrm{~mm}$ in the PTA group and $66 \mathrm{~mm}$ in the DES group. The primary patency rate was $83 \%$ in the DES group compared to $33 \%$ in the PTA group. Primary DES placement was superior to provisional stent placement (primary patency rates of $83 \%$ compared to $73 \%$ ). Provisional DES placement was also superior to provisional BMS placement (90\% vs $73 \%$ primary patency rates). Stent fracture rates were $0.9 \%$ in patients who received DES and BMS.

In the VIBRANT study, Geraghty et al randomized 148 patients with femoropopliteal disease and intermittent claudication or ischemic rest pain to bare nitinol stent implantation or nonheparin-bonded VIABAHN (WL Gore and Associates, Newark, DE, USA) endoprosthesis deployment. The mean lesion length was $188 \mathrm{~mm}$, and $61 \%$ of lesions were occlusive. ${ }^{18}$ The primary patency rate (PSV ratio $\leq 2$ and no target lesion revascularization) at 3 years was similar at $24 \%$ in the nitinol stent group and $26 \%$ in the VIABAHN stent graft group. Fifty percent of nitinol stents had stent fractures compared to only $2.6 \%$ of VIABAHN stent graft group.

In the SUMMIT registry, the EPIC self-expanding nitinol stent (Boston Scientific, Marlborough, MA, USA) was used in 100 patients with femoropopliteal disease. The mean lesion length was $70 \mathrm{~mm}, 29 \%$ of lesions were total occlusions, and $48 \%$ had moderate or severe calcification. ${ }^{19}$ The mean ABI increased from 0.73 to 0.96 in 1 year. The primary patency rate (PSV ratio at 12 months $<2.5 \mathrm{~m} / \mathrm{s}$ ) was $85 \%$. There were no stent fractures reported.

In the COMPLETE SE study, Laird et al implanted the COMPLETE SE laser-cut, self-expanding nitinol stent (Medtronic Vascular, Santa Rosa, CA, USA) in 196 patients with femoropopliteal disease. ${ }^{20}$ A total of $4.2 \%$ had lesions confined to the popliteal artery. The mean lesion length was $61 \mathrm{~mm}$, and moderate-to-severe calcification was noted in $91 \%$ of lesions. The primary patency rate (defined as PSV ratio $<2.0 \mathrm{~m} / \mathrm{s}$ ) was $72.6 \%$ at 1 year, and no stent fractures were detected at 12 months.

The published studies of the Supera interwoven nitinol stent have all been retrospective. However, the results of Supera stents in the femoropopliteal segment appear favorable compared to all other major stent types despite the inclusion of more favorable lesion characteristics (eg, shorter length, calcification, and less popliteal involvement) in other trials (Tables 1 and 2).

\section{Use of Supera in specific lesion types}

Because of its helical design and resistance to compressive forces, the Supera stent may have particular application in otherwise difficult to treat femoropopliteal lesion types, 
including popliteal disease, highly calcified lesions, and long-segment femoropopliteal disease. This section reviews the rationale and data regarding these lesion types and demonstrates representative cases where Supera stents were used clinically.

\section{Popliteal lesions}

Multiple studies have reported on the use of Supera stents in the popliteal artery. In a retrospective study (Leipzig Supera Popliteal Artery Stent Registry) of the use of Supera stents in 101 patients with popliteal arterial disease (38\%, 48\%, and $14 \%$ in P1, P2, and P3 segments, respectively) from 2008 to 2010 at Park Hospital in Leipzig, Germany, ${ }^{21}$ the mean stent length was $84 \mathrm{~mm}$ and the 12-month primary patency rate was $88 \%$. The mean $\mathrm{ABI}$ increased from 0.58 to 0.97 , and the mean Rutherford-Becker classification decreased from 3.1 to 1.4. No stent fractures were found on follow-up.

In the Leipzig Supera 500 registry of the use of Supera stents in femoropopliteal disease, ${ }^{12}$ involving 439 patients treated with Supera stents, $42 \%$ of lesions treated involved the popliteal artery $(13 \%, 18 \%$, and $10 \%$ in P1, P2, and P3 segments, respectively), primary patency rates for the overall group at 12 months and 24 months were $81 \%$ and $62 \%$, respectively, and no stent fractures were reported. Primary patency rates were not different between Supera stents in the SFA and popliteal lesions.

Brescia et al treated 48 patients with femoropopliteal lesions with a mean lesion length of $240 \mathrm{~mm} .{ }^{22}$ In all, $69 \%$ of lesions extended into the popliteal artery from the distal SFA. The primary patency in stents placed in the SFA alone was $89 \%$ versus $75 \%$ for stents extending into the popliteal artery. This difference was not statistically significant $(P=0.17)$.
Chan et al placed Supera stents in 78 patients, out of which, in $54 \%$ of patients, the lesion extended into the aboveknee popliteal artery. In $10 \%$ of patients, the lesion extended into the below-knee popliteal artery. The mean length of the lesions that extended into the popliteal artery was $139 \mathrm{~mm} .{ }^{14}$ One-year primary patency was similar at $79 \%$ in the whole cohort and $73 \%$ among cases in which the lesion extended into the popliteal artery. There were no stent fractures in this study.

Goltz et al reported on 40 patients with Supera stent implantation in the P1 and P2 segments of the popliteal artery from February 2009 to March 2011 at Julius Maximilians University of Würzburg in Germany. ${ }^{23}$ The mean ABI increased from 0.37 to 0.91 , the primary patency rate at 1 year was $68 \%$, and there were no stent fractures.

León et al reported on the use of Supera stents in the popliteal artery in 34 patients with a mean stent length of $119 \mathrm{~mm}$ from February 2008 to December $2010 .{ }^{24}$ In 59\% of cases, the stented lesion extended into the below-knee segment, in $36 \%$ in the above-knee segment, and in $6 \%$ in the behind-knee segment of the popliteal artery. Mean ABI increased from 0.6 to 1.1 . The primary patency rate was $79 \%$, and no stent fractures were detected on follow-up.

Based on the results of the above studies, Supera stents have shown excellent overall patency in this difficult to treat anatomic segment. Figure 1 demonstrates an example of the use of Supera stent in popliteal location in a 63-year-old patient who presented with nonhealing right foot wound and a focal popliteal occlusion.

\section{Heavily calcified lesions}

A number of studies have reported the use of Supera stents in heavily calcified lesions. In the Leipzig Supera
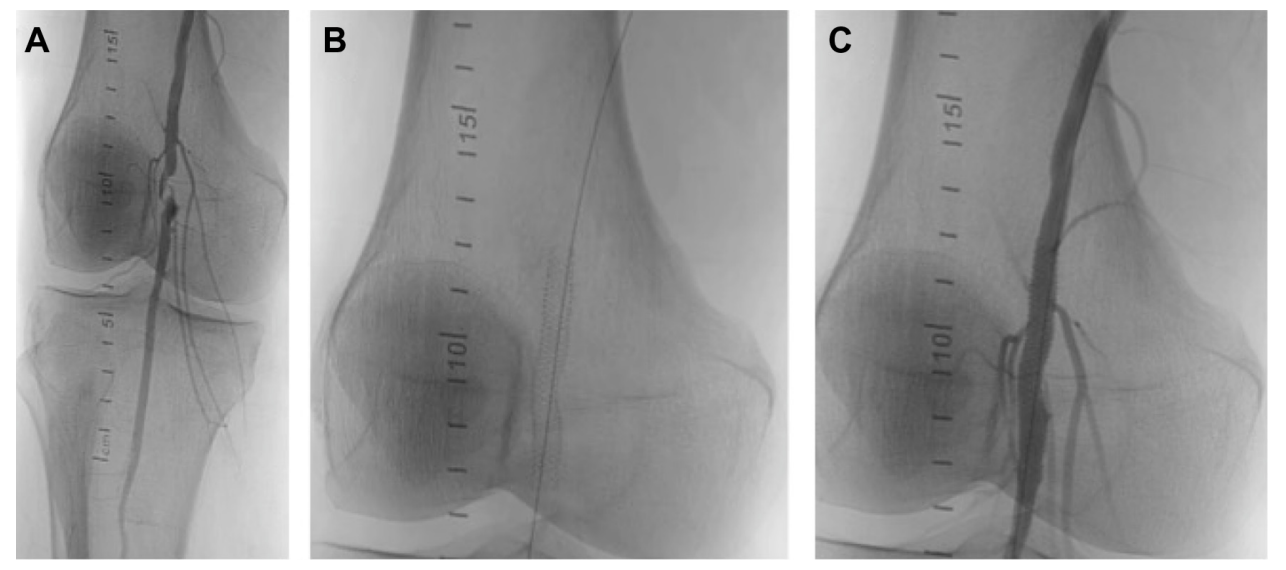

Figure I Focal chronic occlusion in an above-knee segment of the popliteal artery $(\mathbf{A})$ treated with a $5.5 \times 40$ mm Supera stent $(\mathbf{B})$ without significant residual stenosis $(\mathbf{C})$. 

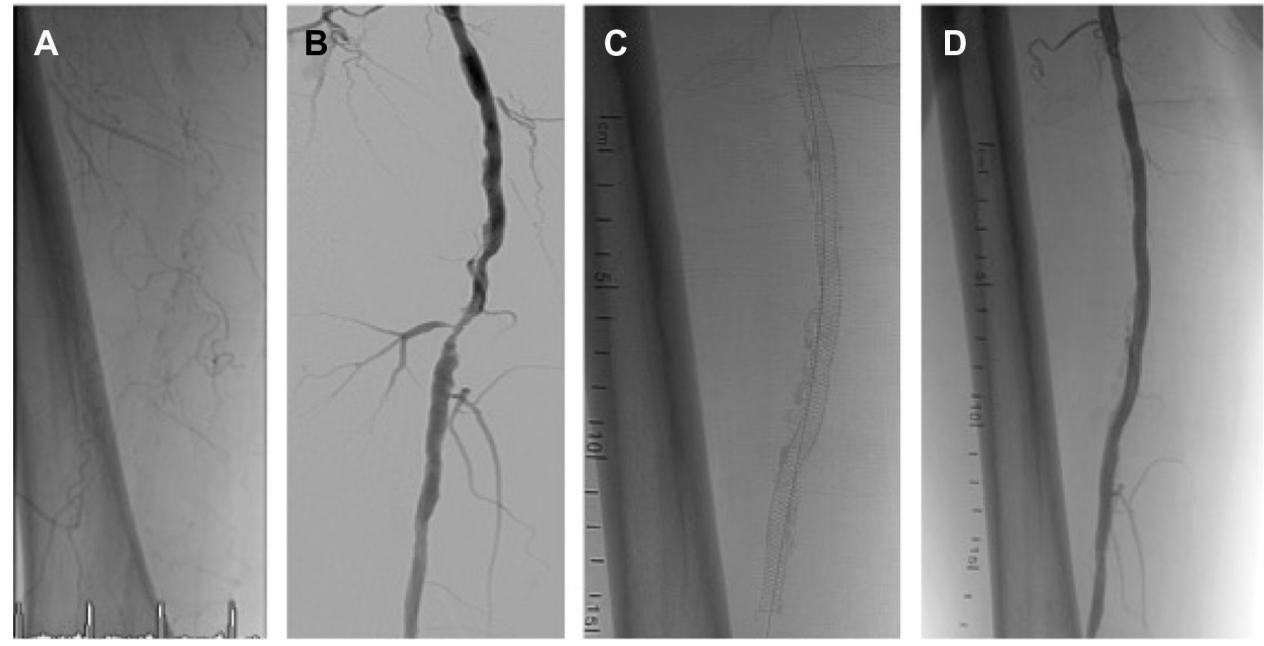

Figure 2 Moderately calcified right SFA (A) with severe stenosis in the middle segment (digital subtraction angiography) (B) treated with a $5.5 \times 150$ mm Supera stent $(\mathbf{C})$ with no significant residual stenosis (D).

Abbreviation: SFA, superficial femoral artery.

500 registry of the use of Supera stents in femoropopliteal disease, ${ }^{12}$ among 439 patients treated with Supera stents, $22 \%$ of lesions treated had moderate and $31 \%$ had severe calcification. While there has not been a dedicated report of an analysis to detect difference in primary patency rates based on severity of calcification, the relatively high 1-year primary patency rate of $81 \%$ suggests that the increased radial strength of the Supera stent may make it suitable for treating highly calcified lesions.

Figure 2 demonstrates an example of the use of the Supera stent for the treatment of a moderate-to-severely calcified SFA lesion with severe stenosis in a 62-year-old smoker who presented with severe lifestyle-limiting claudication. Figure 3 demonstrates the use of a Supera stent for the treatment of severe proximal and mid SFA calcification in an 82-year-old male with severe lifestyle-limiting claudication. In each case, the lesions were predilated with $6 \mathrm{~mm}$ balloons prior to stent deployment, in order to maximize stent expansion and limit elongation.

\section{Long lesions}

A number of studies have evaluated the use of the Supera stent in long femoropopliteal lesions. In the SUPERB study, ${ }^{11} 87$ patients had lesions $<55 \mathrm{~mm}, 88$ patients had lesions $56-92 \mathrm{~mm}$, and 87 patients had lesions 92-236 mm. ${ }^{9}$ The primary patency at 1 year was $85 \%, 78 \%$, and $77 \%$ in the lesions measuring $<55 \mathrm{~mm}, 56-92 \mathrm{~mm}$, and 92-236 mm, respectively. During deployment of a
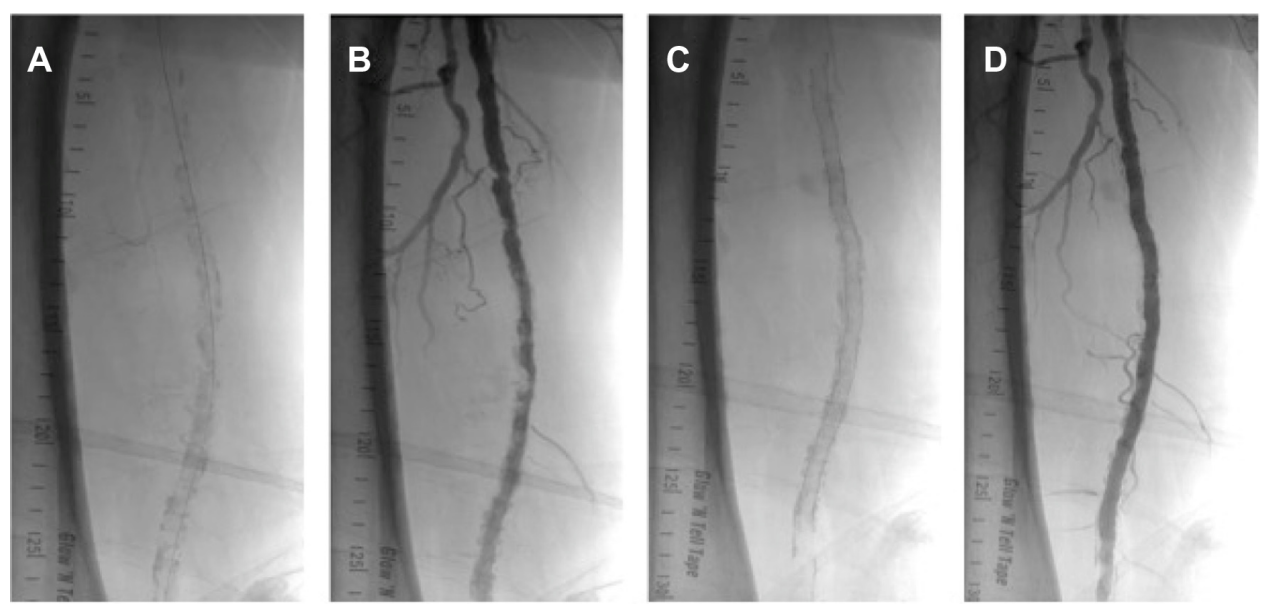

Figure 3 Severely calcified right SFA (A) with severe stenosis in the proximal and middle segment (B) treated with overlapping $5.5 \times 150 \mathrm{~mm}$ and $5.5 \times 60 \mathrm{~mm}$ Supera stents (C) with no significant residual stenosis (D).

Abbreviation: SFA, superficial femoral artery. 


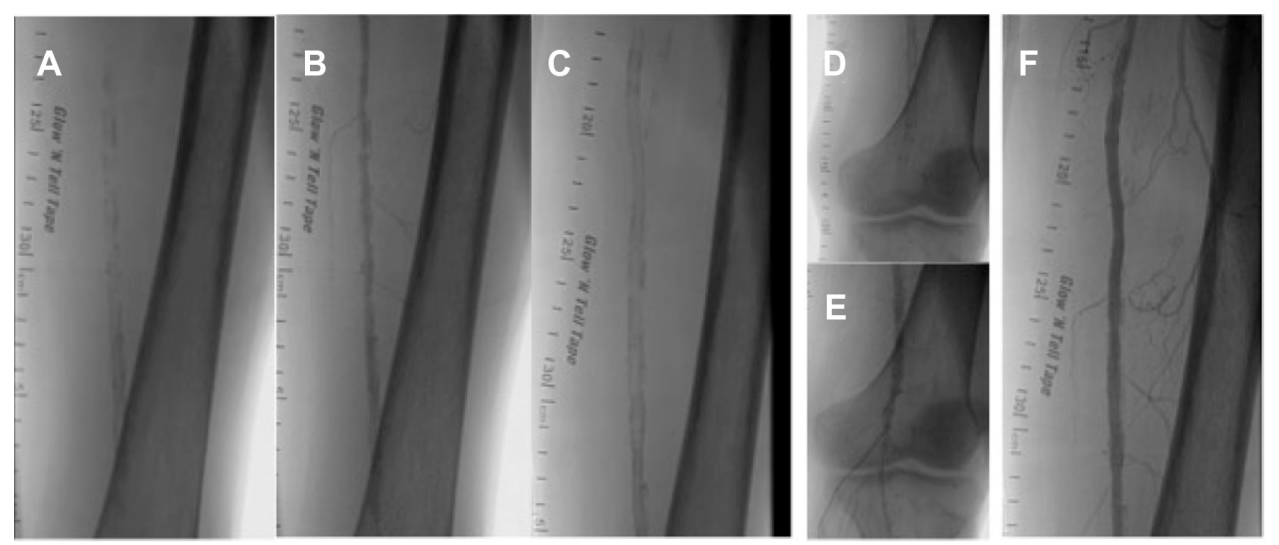

Figure 4 Severely calcified left SFA with an prior distal SFA stent (A) with severe calcification and stenosis proximal (mid SFA) and distal (popliteal above knee) to the previous stent segment (B) treated with overlapping $5.5 \times 150 \mathrm{~mm}$ Supera stent proximal to the old stent (C) and a $5 \times 100 \mathrm{~mm}$ Supera stent distal to the old stent (D) with no significant residual stenosis ( $\mathbf{E}$ and $\mathbf{F}$ ).

Abbreviation: SFA, superficial femoral artery.

Supera stent in a long lesion, particular attention should be paid to the nominal length of the stent. The most optimal patency is observed when the deployed stent length is the same as the labeled (nominal) stent length. Patients with the most severe stent elongation (deployed stent length greater than labeled stent length by more than 40\%) had relatively poor 1 -year primary patency (57\%) compared to a 91\% 1-year patency for stents deployed at nominal length $(P$-value $<0.001)$.

One study in which particularly long lesions were studied suggests no difference in stent patency according to the lesion length. Brescia et al treated 48 patients with femoropopliteal lesions with a mean lesion length of $240 \mathrm{~mm} .^{22}$ At a mean follow-up of 27.5 months, the primary patency rate was $80 \%$. No stent fractures were found in patients who had subsequent procedures. In this study, there were 18 lesions with length $>300 \mathrm{~mm}, 18$ lesions between $150 \mathrm{~mm}$ and $300 \mathrm{~mm}$, and 18 lesions were $<150 \mathrm{~mm}$ in length. There was no difference in primary patency between lesions $>300 \mathrm{~mm}$, lesions $<150 \mathrm{~mm}$, and lesions $150-300 \mathrm{~mm}$ in length. Of note, the operators from this study were particularly careful to avoid stent elongation during deployment. These results suggest that the Supera stent platform may be safely used in longer lesion segments without significant compromise of patency, especially if attention is paid to achieving nominal stent length at deployment.

Figure 4 shows an illustrative case of a 68-year-old male smoker with a previous distal SFA Supera $(4.0 \times 40 \mathrm{~mm})$ 2 years prior who subsequently presented with severe lifestyle-limiting left-calf claudication. He was found to have a long segment of severe calcification and stenosis primarily proximal and distal to the stent with moderate in-stent restenosis. Following predilatation with a $6.0 \mathrm{~mm}$ Angiosculpt balloon, a $5.5 \times 100 \mathrm{~mm}$ Supera stent was deployed distal to the previous stent with overlap and a $5.5 \times 150 \mathrm{~mm}$ Supera stent was deployed proximal to the previous stent with overlap.

\section{Limitations}

While the available data on the use of Supera stents for atherosclerotic femoropopliteal disease is promising, it is mostly gathered from the retrospective studies of medium-length lesions and short-term follow-up. In addition, there has been no direct comparison of the Supera stent platform with angioplasty alone or with other stent designs. Additional studies are needed that include long-term follow-up, inclusion of more complex lesion characteristics (eg, long lesions), and direct comparison with angioplasty alone (including newly available drug-coated balloons), as well as other stent platforms.

\section{Conclusion}

Femoropopliteal artery stenting is an increasingly viable option in the treatment of disabling claudication or limb ischemia. While there have been no direct comparisons of the Supera interwoven nitinol stent system with angioplasty, bare metal stents, or other nitinol stent systems, historical comparison across a diverse range of lesion lengths and complexity suggests that the Supera system may offer improved patency in association with reduced stent fracture rates. Further studies, including randomized controlled studies, comparing the Supera interwoven nitinol stent system with other stent platforms and angioplasty alone are needed. 


\section{Disclosure}

EJA is a consultant for Abbott Vascular, Medtronic, and Spectranetics. KB reports no conflicts of interest in this work.

\section{References}

1. Hirsch AT, Haskal ZJ, Hertzer NR, et al; American Association for Vascular Surgery, Society for Vascular Surgery, Society for Cardiovascular Angiography and Interventions, Society for Vascular Medicine and Biology, Society of Interventional Radiology, ACC/AHA Task Force on Practice Guidelines, American Association of Cardiovascular and Pulmonary Rehabilitation, National Heart, Lung, and Blood Institute, Society for Vascular Nursing, TransAtlantic Inter-Society Consensus, Vascular Disease Foundation. ACC/AHA 2005 practice guidelines for the management of patients with peripheral arterial disease (lower extremity, renal, mesenteric, and abdominal aortic): a collaborative report from the American Association for Vascular Surgery/Society for Vascular Surgery, Society for Cardiovascular Angiography and Interventions, Society for Vascular Medicine and Biology, Society of Interventional Radiology, and the ACC/AHA Task Force on Practice Guidelines (Writing Committee to Develop Guidelines for the Management of Patients With Peripheral Arterial Disease): endorsed by the American Association of Cardiovascular and Pulmonary Rehabilitation; National Heart, Lung, and Blood Institute; Society for Vascular Nursing; TransAtlantic Inter-Society Consensus; and Vascular Disease Foundation. J Am Coll Cardiol. 2006;113(11):e463-e654.

2. Rooke TW, Hirsch AT, Misra S, et al; Society for Cardiovascular Angiography and Interventions, Society of Interventional Radiology, Society for Vascular Medicine, Society for Vascular Surgery. 2011 ACCF/AHA focused update of the guideline for the management of patients with peripheral artery disease (updating the 2005 guideline): a report of the American College of Cardiology Foundation/American Heart Association Task Force on Practice Guidelines. J Am Coll Cardiol. 2011;58(19):2020-2045.

3. European Stroke Organisation, Tendera M, Aboyans V, et al; ESC Committee for Practice Guidelines. ESC guidelines on the diagnosis and treatment of peripheral artery diseases: document covering atherosclerotic disease of extracranial carotid and vertebral, mesenteric, renal, upper and lower extremity arteries: the Task Force on the Diagnosis and Treatment of Peripheral Artery Diseases of the European Society of Cardiology (ESC). Eur Heart J. 2011;32(22):2851-2906.

4. Goodney PP, Beck AW, Nagle J, Welch HG, Zwolak RM. National trends in lower extremity bypass surgery, endovascular interventions, and major amputations. J Vasc Surg. 2009;50(1):54-60.

5. Scheinert D, Scheinert S, Sax J, et al. Prevalence and clinical impact of stent fractures after femoropopliteal stenting. J Am Coll Cardiol. 2005;45(2):312-315.

6. Bosiers M, Torsello G, Gissler HM, et al. Nitinol stent implantation in long superficial femoral artery lesions: 12-month results of the DURABILITY I study. $J$ Endovasc Ther. 2009;16(3):261-269.

7. Bosiers M, Deloose K, Callaert J, et al. Results of the Protégé EverFlex 200-mm-long nitinol stent (ev3) in TASC C and D femoropopliteal lesions. J Vasc Surg. 2011;54(4):1042-1050.

8. Davaine JM, Azéma L, Guyomarch B, et al. One-year clinical outcome after primary stenting for Trans-Atlantic Inter-Society Consensus (TASC) $\mathrm{C}$ and D femoropopliteal lesions (the STELLA "STEnting Long de L'Artère fémorale superficielle" cohort). Eur J Vasc Endovasc Surg. 2012;44(4):432-441.
9. FDA. Supera Peripheral Stent System: Instruction for use. Available from: http://www.accessdata.fda.gov/cdrh_docs/pdf12/P120020b.pdf. Accessed May 21, 2015.

10. Scheinert D, Grummt L, Piorkowski M, et al. A novel self-expanding interwoven nitinol stent for complex femoropopliteal lesions: 24-month results of the SUPERA SFA registry. J Endovasc Ther. 2011;18(6): 745-752.

11. Garcia L, Jaff MR, Metzger C, et al; SUPERB Trial Investigators*. Wire-interwoven nitinol stent outcome in the superficial femoral and proximal popliteal arteries: twelve-month results of the SUPERB trial. Circ Cardiovasc Interv. 2015;8(5):e000937.

12. Werner M, Paetzold A, Banning-Eichenseer U, et al. Treatment of complex atherosclerotic femoropopliteal artery disease with a selfexpanding interwoven nitinol stent: midterm results from the Leipzig SUPERA 500 registry. EuroIntervention. 2014;10(7):861-868.

13. Dumantepe M, Seren M, Fazliogullari O, Ayoglu U, Teymen B. Treatment of complex atherosclerotic femoropopliteal artery disease with a selfexpanding interwoven nitinol stent: midterm results. Vascular. 2015. Epub ahead of print.

14. Chan YC, Cheng SW, Ting AC, Cheung GC. Primary stenting of femoropopliteal atherosclerotic lesions using new helical interwoven nitinol stents. J Vasc Surg. 2014;59(2):384-391.

15. George JC, Rosen ES, Nachtigall J, VanHise A, Kovach R. SUPERA interwoven nitinol stent outcomes in above-knee IntErventions (SAKE) study. J Vasc Interv Radiol. 2014;25(6):954-961.

16. Laird JR, Katzen BT, Scheinert D, et al; RESILIENT Investigators. Nitinol stent implantation versus balloon angioplasty for lesions in the superficial femoral artery and proximal popliteal artery: twelve-month results from the RESILIENT randomized trial. Circ Cardiovasc Interv. 2010;3(3):267-276.

17. Dake MD, Ansel GM, Jaff MR, et al; Zilver PTX Investigators. Paclitaxel-eluting stents show superiority to balloon angioplasty and bare metal stents in femoropopliteal disease: twelve-month Zilver PTX randomized study results. Circ Cardiovasc Interv. 2011;4(5):495-504.

18. Geraghty PJ, Mewissen MW, Jaff MR, Ansel GM; VIBRANT Investigators. Three-year results of the VIBRANT trial of VIABAHN endoprosthesis versus bare nitinol stent implantation for complex superficial femoral artery occlusive disease. J Vasc Surg. 2013;58(2):386-395. e4.

19. Werner M, Piorkowski M, Thieme M, et al. SUMMIT registry: one-year outcomes after implantation of the EPIC self-expanding nitinol stent in the femoropopliteal segment. J Endovasc Ther. 2013;20(6):759-766.

20. Laird JR, Jain A, Zeller T, et al; Complete SE Investigators. Nitinol stent implantation in the superficial femoral artery and proximal popliteal artery: twelve-month results from the complete SE multicenter trial. J Endovasc Ther. 2014;21(2):202-212.

21. Scheinert D, Werner M, Scheinert S, et al. Treatment of complex atherosclerotic popliteal artery disease with a new self-expanding interwoven nitinol stent. JACC Cardiovasc Interv. 2013;6(1):65-71.

22. Brescia AA, Wickers BM, Correa JC, Smeds MR, Jacobs DL. Stenting of femoropopliteal lesions using interwoven nitinol stents. J Vasc Surg. 2015. 61(6): 1472-1478.

23. Goltz JP, Ritter CO, Kellersmann R, Klein D, Hahn D, Kickuth R. Endovascular treatment of popliteal artery segments $\mathrm{P} 1$ and $\mathrm{P} 2$ in patients with critical limb ischemia: initial experience using a helical nitinol stent with increased radial force. J Endovasc Ther. 2012;19(3):450-456.

24. León LR Jr, Dieter RS, Gadd CL, et al. Preliminary results of the initial United States experience with the Supera woven nitinol stent in the popliteal artery. J Vasc Surg. 2013;57(4):1014-1022. 
Vascular Health and Risk Management

Dovepress

\section{Publish your work in this journal}

Vascular Health and Risk Management is an international, peerreviewed journal of therapeutics and risk management, focusing on concise rapid reporting of clinical studies on the processes involved in the maintenance of vascular health; the monitoring, prevention and treatment of vascular disease and its sequelae; and the involvement of metabolic disorders, particularly diabetes. This journal is indexed on PubMed Central and MedLine. The manuscript management system is completely online and includes a very quick and fair peer-review system, which is all easy to use. Visit http://www.dovepress.com/ testimonials.php to read real quotes from published authors.

Submit your manuscript here: http://www.dovepress.com/vascular-health-and-risk-management-journal 